IJMMS 2004:9, 479-485

PII. S0161171204208274

http://ijmms.hindawi.com

(C) Hindawi Publishing Corp.

\title{
INTERPOLATION METHODS TO ESTIMATE EIGENVALUE DISTRIBUTION OF SOME INTEGRAL OPERATORS
}

\author{
E. M. EL-SHOBAKY, N. ABDEL-MOTTALEB, A. FATHI, \\ and M. FARAGALLAH
}

Received 20 August 2002

\begin{abstract}
We study the asymptotic distribution of eigenvalues of integral operators $T_{k}$ defined by kernels $k$ which belong to Triebel-Lizorkin function space $F_{p u}^{\sigma}\left(F_{q v}^{\tau}\right)$ by using the factorization theorem and the Weyl numbers $x_{n}$. We use the relation between Triebel-Lizorkin space $F_{p u}^{\sigma}(\Omega)$ and Besov space $B_{p q}^{\tau}(\Omega)$ and the interpolation methods to get an estimation for the distribution of eigenvalues in Lizorkin spaces $F_{p u}^{\sigma}\left(F_{q v}^{\tau}\right)$.
\end{abstract}

2000 Mathematics Subject Classification: 46B20, 47B10.

1. Lizorkin kernels. We will use the following notation: $l_{p, q}, S_{p q}^{(x)}, B_{p q}^{s}$, and $F_{p q}^{s}$ to denote Lorentz sequence space, Schatten class, Besov function space, and Triebel-Lizorkin function space, respectively. By $\pi_{p}, s_{n}$, and $x_{n}$ we denote $p$-summing norms, $s$-number function, and Weyl numbers, respectively, see [2, 4, 5].

THEOREM 1.1 (see [1]). Let $s \in \mathbb{R}, p \in(0, \infty)$ and let $q, u, v \in(0, \infty]$. Then $B_{p u}^{s} \subset F_{p q}^{s} \subset$ $B_{p v}^{s}$ if and only if

$$
0<u \leq \min (p, q), \quad \max (p, q) \leq v \leq \infty .
$$

That is, if and only if $0<u \leq q \leq v$.

Proposition 1.2 (see [4]). Let $\Phi \in\left[B_{p u}^{\sigma}(0,1), X\right]$ and $r=\max (p, u)$. Then

$$
\Phi_{\text {op }}: a \longrightarrow(\Phi(\cdot), a)
$$

(where $\Phi_{\mathrm{op}}$ is an approximable operator from $X^{\prime}$ into $\left.B_{p u}^{\sigma}(0,1)\right)$ defines an absolutely $r$-summing operator from $X^{\prime}$ into $B_{p u}^{\sigma}(0,1)$. Moreover,

$$
\left\|\Phi_{\text {op }}\left|\pi_{r}\|\leq\| \Phi\right|\left[B_{p u}^{\sigma}, X\right]\right\| .
$$

We restate the previous proposition in the following form in the case of TriebelLizorkin space $F_{p u}^{\sigma}(\Omega)$.

Proposition 1.3. Let $X$ be a Banach space, $\Omega \subset \mathbb{R}^{N}$ a bounded domain, $\sigma>0$, and $1 \leq p<\infty$. Let $\Phi \in F_{p u}^{\sigma}(\Omega ; X)$ and $r=\max (p, u)$. Then

$$
\Phi_{\mathrm{op}}: x \longrightarrow(\Phi(\cdot), x)
$$


defines an absolutely $r$-summing operator from $X^{\prime}$ into $F_{p u}^{\sigma}(X)$. Moreover,

$$
\pi_{r}\left(\Phi_{\mathrm{op}}\right) \leq\|\Phi\|_{p, u, \sigma ; \Omega, X}
$$

Proof. Given $x_{1}, \ldots, x_{n} \in X^{\prime}$, Jessen's inequality [4] yields

$$
\left(\int_{\Omega}\left[\sum_{i=1}^{n}\left|\left(\Phi(\xi), x_{i}\right)\right|^{r}\right]^{p / r} d \xi\right)^{1 / p} \leq\left(\sum_{i=1}^{n}\left[\int_{\Omega}\left|\left(\Phi(\xi), x_{i}\right)\right|^{p} d \xi\right]^{r / p}\right)^{1 / r}
$$

Therefore,

$$
\left\|\left(\sum_{i=1}^{n}\left|\left(\Phi(\cdot), x_{i}\right)\right|^{r}\right)^{1 / r}\right\|_{L_{p}} \leq\left(\sum_{i=1}^{n}\left\|\left(\Phi(\cdot), x_{i}\right)\right\|_{L_{p}}^{r}\right)^{1 / r} \leq\|\Phi\|_{L_{p}}\left\|\left(x_{i}\right)\right\|_{\pi_{r}} .
$$

Applying this result to $\Delta_{\tau}^{m} \Phi$, we obtain

$$
\left\|\left(\sum_{i=1}^{n}\left|\left(\Delta_{\tau}^{m} \Phi(\cdot), x_{i}\right)\right|^{r}\right)^{1 / r}\right\|_{L_{p}} \leq\left\|\Delta_{\tau}^{m} \Phi\right\|_{L_{p}}\left\|\left(x_{i}\right)\right\|_{\pi_{r}} .
$$

Hence,

$$
\begin{gathered}
{\left[\int_{\Omega}\left(\sum_{i=1}^{n}\left[\tau^{-\sigma}\left\|\left(\Delta_{\tau}^{m} \Phi(\cdot), x_{i}\right)\right\|_{L_{p}}\right]^{r}\right)^{u / r} \frac{d \tau}{\tau}\right]^{1 / u}} \\
\leq\left[\sum_{i=1}^{n}\left(\int_{\Omega}\left[\tau^{-\sigma}\left\|\Delta_{\tau}^{m} \Phi(\cdot), x_{i}\right\|_{L_{p}}\right]^{u}\right)^{r / u}\right]^{1 / r} \\
\leq\left\|\left(\left[\int_{\Omega} \tau^{-\sigma}\left|\Delta_{\tau}^{m} \Phi\right|\right]^{u} \frac{d \tau}{\tau}\right)^{1 / u}\right\|_{L_{p}}\left\|\left(x_{i}\right)\right\|_{\pi_{r}} .
\end{gathered}
$$

Finally, we conclude from the preceding inequalities that

$$
\begin{aligned}
& \left\|\left(\sum_{i=1}^{n}\left|\left(\Phi(\cdot), x_{i}\right)\right|^{r}\right)^{1 / r}\right\|_{F_{p u}^{\sigma}} \\
& \quad \leq\left\|\left(\sum_{i=1}^{n}\left|\left(\Phi(\cdot), x_{i}\right)\right|^{r}\right)^{1 / r}\right\|_{L_{p}}+\left[\int_{\Omega}\left(\sum_{i=1}^{n}\left[\tau^{-\sigma}\left\|\left(\Delta_{\tau}^{m} \Phi(\cdot), x_{i}\right)\right\|_{L_{p}}\right]^{r}\right)^{u / r} \frac{d \tau}{\tau}\right]^{1 / u} \\
& \quad \leq\left(\|\Phi\|_{L_{p}}+\left\|\left(\left[\int_{\Omega} \tau^{-\sigma}\left|\Delta_{\tau}^{m} \Phi\right|\right]^{u} \frac{d \tau}{\tau}\right)^{1 / u}\right\|_{L_{p}}\right)=\|\Phi\|_{F_{p u}^{\sigma}}\left\|\left(x_{i}\right)\right\|_{\pi_{r}} .
\end{aligned}
$$

This shows that $\Phi_{\mathrm{op}}$ is absolutely $r$-summing. 
Corollary 1.4 (see [2]). Let $X$ and $Y$ be Banach spaces, $2 \leq p<\infty$, and $T \in \pi_{p, 2}(X, Y)$. Then $T \in S_{p, \infty}^{x}(X, Y)$, and for any $n \in \mathbb{N}$,

$$
x_{n}(T) \leq n^{-1 / p} \pi_{p, 2}(T) .
$$

We are interested in the following theorem.

TheOREM 1.5 (see [3]). Let $1 \leq p \leq \max (2, q) \leq \infty$. Then

$$
x_{n}\left(I_{p, q}^{m}: l_{p}^{m} \rightarrow l_{q}^{m}\right) \asymp \begin{cases}n^{1 / q-1 / p} & \text { for } 1 \leq p \leq q \leq 2, \\ n^{1 / 2-1 / p} & \text { for } 1 \leq p \leq 2 \leq q<\infty, \\ 1 & \text { for } 2 \leq p \leq q<\infty .\end{cases}
$$

THEOREM 1.6 ((multiplication theorem) [4]). If $1 / p+1 / q=1 / r$ and $1 / u+1 / v=1 / w$, then

$$
S_{p u}^{(x)} \circ S_{q v}^{(x)} \subseteq S_{r w}^{(x)}
$$

Theorem 1.7 ((eigenvalue theorem) [2]). Let $0<p<\infty, 0<q \leq \infty$, and let $X$ be $a$ Banach space. Then any operator $T \in L(X)$ which has Weyl numbers $\left(x_{n}(T)\right) \in l_{p, q}$, $T \in S_{p, q}^{(x)}(X)$ is a Riesz operator, the eigenvalue sequence of which is in $l_{p, q}$, and the following inequality holds

$$
\left\|\left(\lambda_{n}(T)\right)\right\|_{p, q} \leq c\left\|\left(x_{n}(T)\right)\right\|_{p, q} .
$$

2. Eigenvalue theorem for Lizorkin kernels. The following theorem contains the main result of this note.

THEOREM 2.1. Let $\Omega \subset \mathbb{R}^{N}$ be a bounded domain, $1 \leq p, q, u, v<\infty$, and $\sigma+\tau>$ $N(1 / p+1 / q-1)$. Define $r$ by $1 / r=(\sigma+\tau) / N+1 / q^{+}$, where $q^{+}=\max \left(q^{\prime}, 2\right)$. Then the eigenvalues of any kernel $k \in F_{p u}^{\sigma}\left(\Omega ; F_{q v}^{\tau}(\Omega)\right)$ belong to the Lorentz sequence space $l_{r, p}$ with

$$
\left\|\left(\lambda_{n}(k)\right)_{n \in \mathbb{N}}\right\|_{l_{r, p}} \leq c\|k\|_{F_{p u}^{\sigma}\left(F_{q v}^{\tau}\right)} .
$$

The constant $c$ depends only on the indices and $\Omega$.

Proof. First, we assume that $p \leq q^{\prime}$.

We will show that there exists an imbedding map id : $F_{p u}^{\sigma}(\Omega) \hookrightarrow F_{q v}^{\tau}(\Omega)^{\prime}$ and then estimate its Weyl numbers $x_{n}(\mathrm{id})$. We factories an imbedding map id : $F_{p u}^{\sigma}(\Omega) \hookrightarrow F_{q v}^{\tau}(\Omega)^{\prime}$ with the help of maps $A$ and $B$ such that

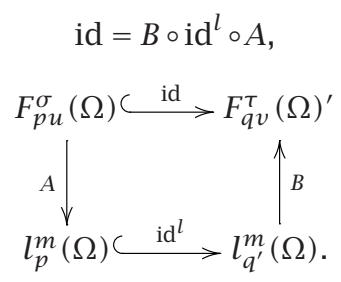


This means that

$$
x_{n}(\mathrm{id}) \leq\|A\| x_{n}\left(\operatorname{id}^{l}\right)\|B\|
$$

if we are able to estimate $\|A\|$ and $\|B\|$ suitably; from [6], operators $A$ and $B$ are defined exactly as they are in [4], and assume that $\Omega$ contains the unit cube in $\mathbb{R}^{N}$ and divide the unit cube in the usual way into $2^{j N}$ congruent cubes with side length $2^{-j}$.

From [1], we have

$$
\|A\| \leq c_{1} 2^{-j(\sigma-N / p)}, \quad\|B\| \leq c_{2} 2^{j\left(-\tau-N / q^{\prime}\right)} .
$$

Substituting (2.4) in (2.3), we get

$$
x_{n}(\mathrm{id}) \leq c 2^{-j(\sigma+\tau)+j N\left(1 / p-1 / q^{\prime}\right)} x_{n}\left(\operatorname{id}^{l}\right) .
$$

By Theorem 1.5, we have

$$
x_{n}\left(\mathrm{id}: F_{p u}^{\sigma}(\Omega) \hookrightarrow F_{q v}^{\tau}(\Omega)^{\prime}\right)<n^{-\rho},
$$

where

$$
\rho=\frac{\sigma+\tau}{N}+ \begin{cases}0, & \text { if } 1 \leq p \leq q^{\prime} \leq 2, \\ \frac{1}{2}-\frac{1}{q}, & \text { if } 1 \leq p \leq 2 \leq q^{\prime}<\infty, \\ 1-\frac{1}{p}-\frac{1}{q}, & \text { if } 2 \leq p \leq q^{\prime}<\infty,\end{cases}
$$

and $n=2^{N j}$.

Hence,

$$
\operatorname{id} \in S_{1 / \rho, \infty}^{(x)}\left(F_{p u}^{\sigma}(\Omega) \hookrightarrow F_{q v}^{\tau}(\Omega)^{\prime}\right) .
$$

To estimate the Weyl number of $T_{k}$ in $F_{q v}^{\tau}(\Omega)^{\prime}$, we use the factorization

$$
F_{q v}^{\tau}(\Omega)^{\prime} \stackrel{T_{k}}{\longrightarrow} F_{p u}^{\sigma}(\Omega) \stackrel{\mathrm{id}}{\leftrightarrow} F_{q v}^{\tau}(\Omega)^{\prime} .
$$

By Proposition 1.3, $k \in F_{p u}^{\sigma}(\Omega ; X)$ implies that $T_{k}: X^{\prime} \hookrightarrow F_{p u}^{\sigma}(\Omega)$ is $p$-summing. By Corollary 1.4, we have

$$
x_{n}\left(T_{k}: F_{q v}^{\tau}(\Omega)^{\prime} \longrightarrow F_{p u}^{\sigma}(\Omega)\right) \leq \pi_{p}\left(T_{k}\right) n^{-1 / \max (p, 2)} \leq c_{1}\|k\|_{F_{p u}^{\sigma}(X)} n^{-1 / \max (p, 2)},
$$

that is,

$$
T_{k} \in S_{s, \infty}^{(x)}\left(F_{p u}^{\sigma}(\Omega), F_{q v}^{\tau}(\Omega)^{\prime}\right), \quad s=\max (p, 2) .
$$

We conclude from the multiplication theorem that id $\circ T_{k} \in S_{r, \infty}^{(x)}\left(F_{q v}^{\tau}(\Omega)^{\prime}\right)$, where $1 / r=$ $\rho+1 / s$. 
In the case when $p>q^{\prime}$, then we have

$$
k \in F_{p u}^{\sigma}\left(\Omega ; F_{q v}^{\tau}(\Omega)\right) \Longrightarrow k \in F_{q^{\prime} u}^{\sigma}\left(\Omega ; F_{q v}^{\tau}(\Omega)\right) .
$$

In this way the second case is reduced to the first one.

So, we have shown that the map $k \rightarrow \mathrm{id} \circ T_{k}$, which assigns to every kernel the corresponding operator, acts as follows:

$$
\text { op : } F_{p u}^{\sigma}\left(F_{q v}^{\tau}\right) \longrightarrow S_{r, \infty}^{(x)}\left(F_{q v}^{\tau}(\Omega)^{\prime}\right)
$$

This result can be improved by interpolation. To this end, choose $p_{0}, p_{1}$, and $\theta$ such that $1 / p=1-\theta / p_{0}+\theta / p_{1}$. We now apply the formula

$$
\left(F_{p_{0} u}^{\sigma}(E), F_{p_{1} u}^{\sigma}(E)\right)_{\theta, p}=F_{p u}^{\sigma}(E), \quad E=F_{q v}^{\tau} .
$$

Then, using interpolation as in [2], where $1 / r=1-\theta / r_{0}+\theta / r_{1}$, hence

$$
\left(S_{r_{0} \infty}^{(x)}, S_{r_{1} \infty}^{(x)}\right)_{\theta, p} \subseteq S_{r p}^{(x)}
$$

Hence the interpolation property yields

$$
\text { op : } F_{p u}^{\sigma}\left(F_{q v}^{\tau}\right) \rightarrow S_{r, p}^{(x)}\left(F_{q v}^{\tau}(\Omega)^{\prime}\right)
$$

By the eigenvalue theorem (Theorem 1.7), we therefore obtain $\left(\lambda_{n}(k)\right) \in l_{r, p}$.

THEOREM 2.2 (eigenvalue theorem for Sobolev kernels). Let $1 \leq p<\infty, 1<q<\infty$, $1 / r=m+n+1 / q^{+}$, and $w=\min (q, 2)$.

Then

$$
k \in\left[W_{p}^{m}(0,1), W_{q}^{n}(0,1)\right] \Longrightarrow\left(\lambda_{n}(k)\right) \in l_{r, w} .
$$

Proof. See [4].

The following example proves that our result improves the previous theorem of [4].

THEOREM 2.3. Let $\Omega \subset \mathbb{R}^{N}$ be a bounded domain, $1 \leq p, q, v<\infty$, and $\tau>0$ with $\tau>N(1 / p+1 / q-1), p \leq v$, and $1 / r:=\tau / N+1 / \max \left(2, q^{\prime}\right)$. Then the eigenvalues of any kernel $k \in L_{p}\left(F_{q v}^{\tau}\right)$ belong to the Lorentz sequence space $l_{r, v}$ with

$$
\left\|\left(\lambda_{n}(k)\right)_{n \in N}\right\|_{l_{r, v}} \leq c\|k\|_{L_{p}\left(F_{q v}^{\tau}\right)}
$$

Proof. We may assume that $p \leq q^{\prime}$. Then, reasoning similarly as in the proof of Theorem 2.1, it follows that the map $k \rightarrow T_{k}$, which assigns to every kernel the corresponding operator, acts as follows:

$$
\text { op }: L_{p}\left(F_{q v}^{\tau}\right) \longrightarrow S_{r, \infty}^{(x)}\left(L_{p}(\Omega)\right)
$$


This result can be improved by interpolation. To this end, we apply the imbedding

$$
\left(L_{p},\left(E_{0}, E_{1}\right)_{\theta, m}\right) \subseteq\left(\left(L_{p}, E_{0}\right),\left(L_{p}, E_{1}\right)\right)_{\theta, m}, \quad p<m,
$$

to the interpolation couple $\left(F_{\mathcal{q}, v_{0}}^{\tau_{0}}, F_{\mathcal{q}, v_{1}}^{\tau_{1}}\right)$. The interpolation property now implies that

$$
\text { op }: L_{p}\left(F_{q v}^{\tau}\right) \rightarrow S_{r, v}^{(x)}\left(L_{p}(\Omega)\right) .
$$

By the eigenvalue theorem (Theorem 1.7), we therefore obtain $\left(\lambda_{n}(k)\right) \in l_{r, v}$.

EXAMPLE 2.4. (1) In this example, we will indicate a special case of the Lizorkin space $F_{p u}^{\sigma}\left(\mathbb{R}^{N}\right)$. When $1<p<\infty$ and $s \in \mathbb{N}_{0}$, then

$$
F_{p, 2}^{s}\left(\mathbb{R}^{N}\right)=W_{p}^{s}\left(\mathbb{R}^{N}\right)
$$

are the classical Sobolev spaces.

We compare this case with Theorem 2.2. We find that

$$
k \in W_{p}^{\sigma}\left(W_{q}^{\tau}\right) \Longrightarrow\left(\lambda_{n}(k)\right) \in l_{r, w},
$$

where $w=\min (q, 2)$, and

$$
k \in F_{p u}^{\sigma}\left(F_{q v}^{\tau}\right) \Longrightarrow\left(\lambda_{n}(k)\right) \in l_{r, p}
$$

We conclude that if $p<w=\min (q, 2), 2 \leq q<\infty, 1<p<2$, then

$$
l_{r, w} \subset l_{r, p},
$$

that is,

$$
\left\|\left(\lambda_{n}(k)\right)_{n \in \mathbb{N}}\right\|_{r, p} \leq\left\|\left(\lambda_{n}(k)\right)_{n \in \mathbb{N}}\right\|_{r, w} .
$$

(2) We compare

$$
k \in W_{p}^{\sigma}\left(W_{q}^{\tau}\right) \Longrightarrow\left(\lambda_{n}(k)\right) \in l_{r, w},
$$

where $w=\min (q, 2)$, with

$$
k \in L_{p}\left(F_{q v}^{\tau}\right) \Longrightarrow\left(\lambda_{n}(k)\right) \in l_{r, v},
$$

where $p \leq v$. We conclude that if $v<w=\min (q, 2), 2 \leq q<\infty, 1<p<2$, then

$$
l_{r, w} \subset l_{r, v},
$$

that is,

$$
\left\|\left(\lambda_{n}(k)\right)_{n \in N}\right\|_{r, v} \leq\left\|\left(\lambda_{n}(k)\right)_{n \in N}\right\|_{r, w} .
$$




\section{REFERENCES}

[1] D. E. Edmunds and H. Triebel, Function Spaces, Entropy Numbers, Differential Operators, Cambridge Tracts in Mathematics, vol. 120, Cambridge University Press, Cambridge, 1996.

[2] H. König, Eigenvalue Distribution of Compact Operators, Birkhäuser Verlag, Basel, 1968.

[3] R. Linde, s-numbers of diagonal operators and Besov embeddings, Rend. Circ. Mat. Palermo (2) Suppl. (1985), no. 10, 83-110, Proc. 13th Winter school.

[4] A. Pietsch, Eigenvalues and s-Numbers, Mathematik und ihre Anwendungen in Physik und Technik, vol. 43, Akademische Verlagsgesellschaft Geest and Portig K.-G., Leipzig, 1987.

[5] H. Triebel, Interpolation Theory, Function Spaces, Differential Operators, North-Holland Mathematical Library, vol. 18, North-Holland Publishing, Amsterdam, 1978.

[6] _ Theory of Function Spaces. II, Monographs in Mathematics, vol. 84, Birkhäuser Verlag, Basel, 1992.

E. M. El-Shobaky: Department of Mathematics, Faculty of Science, Ain Shams University, Cairo 11566, Egypt

E-mail address: solar@photoenergy.org

N. Abdel-Mottaleb: Department of Mathematics, Faculty of Science, Ain Shams University, Cairo 11566, Egypt

A. Fathi: Department of Mathematics, Faculty of Science, Ain Shams University, Cairo 11566, Egypt

E-mail address: a_fath72@yahoo.com

M. Faragallah: Department of Mathematics, Faculty of Education, Ain Shams University, Cairo 11566, Egypt 


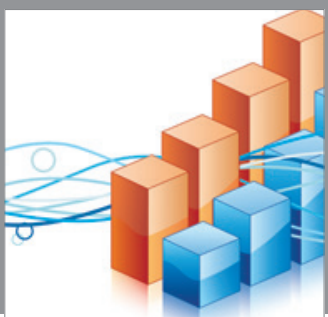

Advances in

Operations Research

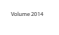

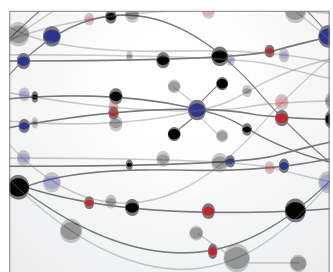

\section{The Scientific} World Journal
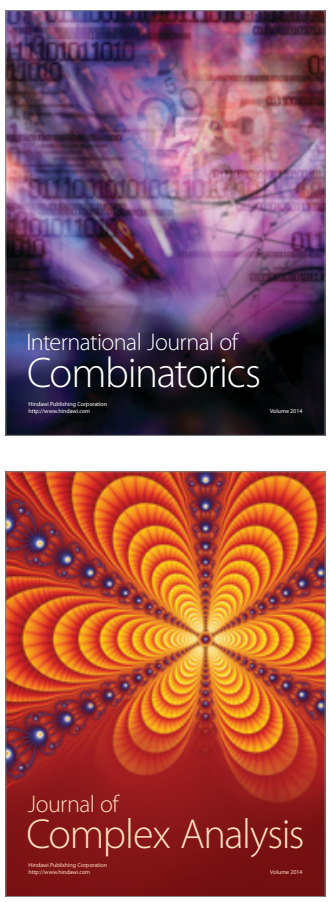

International Journal of

Mathematics and

Mathematical

Sciences
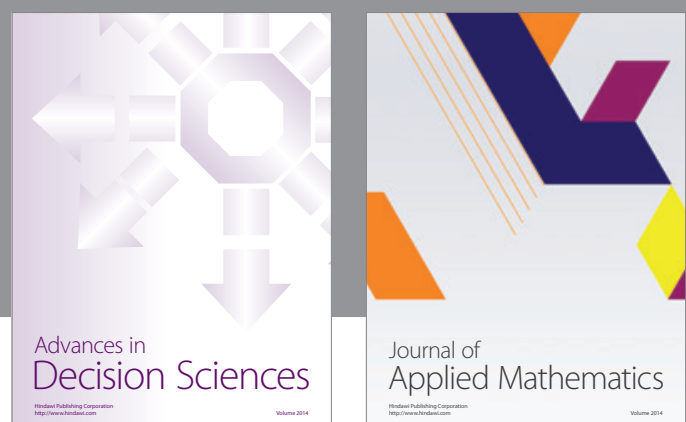

Journal of

Applied Mathematics
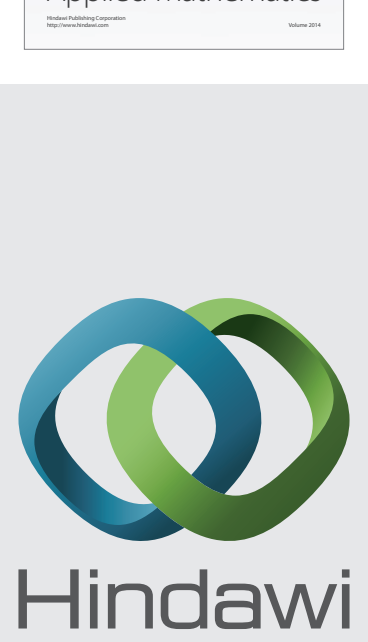

Submit your manuscripts at http://www.hindawi.com
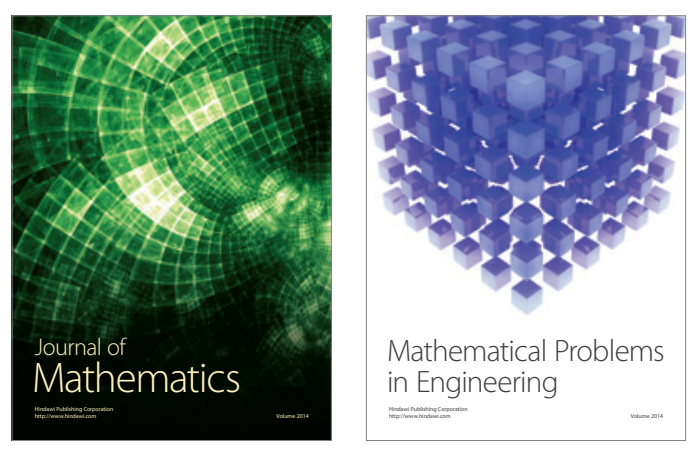

Mathematical Problems in Engineering
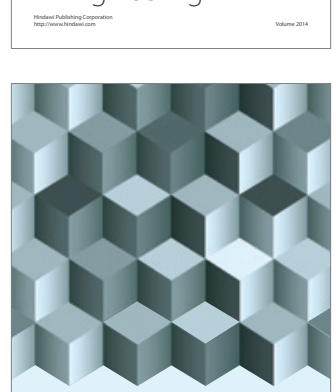

Journal of

Function Spaces
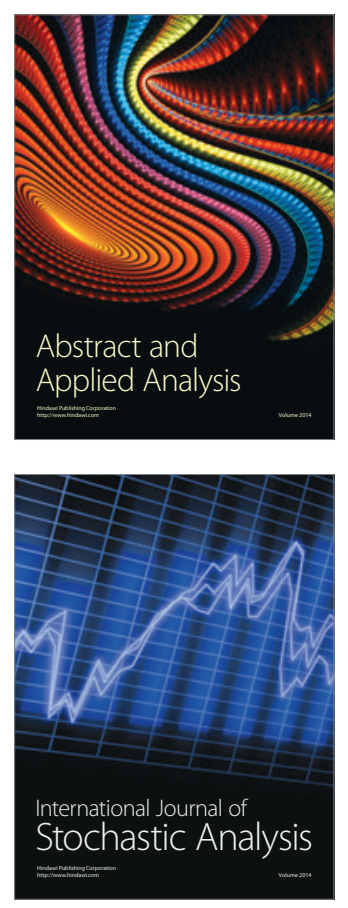

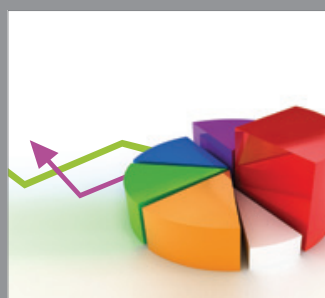

ournal of

Probability and Statistics

Promensencen
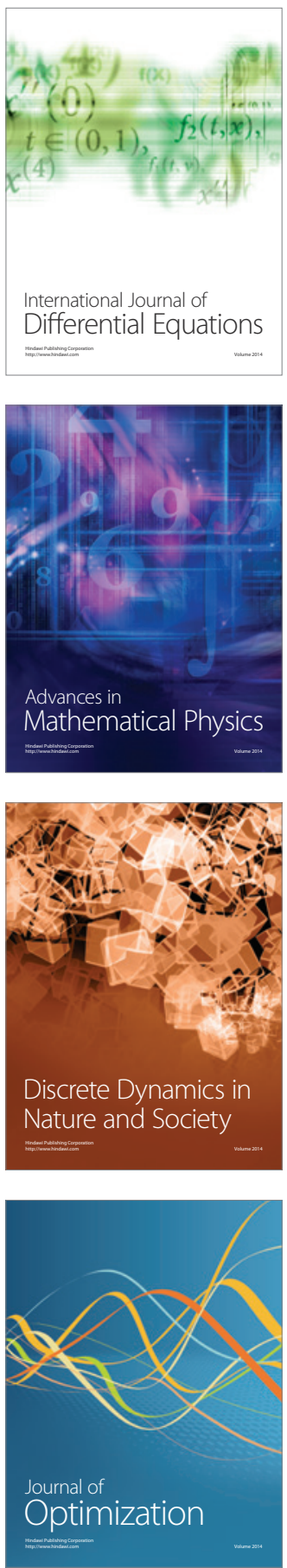